\title{
Fatores de Risco Associados à Osteoporose em uma População de Mulheres Brasileiras Residentes em São José do Rio Pardo, Estado de São Paulo
}

\author{
Risk Factors Associated to Osteoporosis in Brazilian Women from \\ São José do Rio Pardo, São Paulo
}

\author{
Paulo Domingos Parisi Júnior ${ }^{(1)}$, Wiliam Habib Chahade ${ }^{(2)}$
}

\section{RESUMO}

Objetivos: verificar os principais fatores de risco associados à baixa massa óssea e propor algoritmos para a indicação de avaliação da densidade óssea em uma população de mulheres, residentes em São José do Rio Pardo, Estado de São Paulo. Métodos: foram estudadas 324 mulheres com média de idade de 60 anos. Foi enviado pelo correio um questionário contendo perguntas a respeito de diversos fatores de risco para osteoporose, incluindo histórico pessoal e materno de fraturas. A densidade mineral óssea foi medida utilizando-se a densitometria óssea por densitômetro DXA, sendo analisados os sítios coluna lombar (L2-L4) e/ou fềmur proximal (colo femoral); todas as mulheres foram classificadas de acordo com os critérios da OMS. A análise estatística empregou Análise de Variância (Anova), segundo um critério de classificação e teste "post-hoc" de comparação múltipla, aos pares, de Student - Newman - Keuls; tabelas de contingência 2 x 2 e k x r; teste do Qui-quadrado. Por meio de regressões múltiplas foram estabelecidas as variáveis mais eficientes na identificação de mulheres com baixa massa óssea. Foram também aplicados dois algoritmos descritos em literatura, denominados Osteorisk e Osiris. Resultados: o grupo Normal ficou constituído por $88(27,2 \%)$ indivíduos, o grupo Osteopênico por $160(49,4 \%)$ e o grupo Osteoporótico por 76 (23,5\%). A média de idade dos grupos foi: Normal, 56 anos; Osteopenia, 60 anos; e Osteoporose, 65 anos. O peso e a estatura foram, respectivamente, para o grupo Normal 69,5 kg e 1,60 m; Osteopenia, 64,9 kg e 1,59 m; e Osteoporose, 62,1 kg e $1,57 \mathrm{~m}$. Todos esses valores mostraram significância estatística. Outras variáveis que mostraram diferenças significantes foram: número de anos decorridos desde a menopausa, maior no grupo Osteoporose; uso de anticoncepcionais e de terapia de reposição hormonal, que foi menos freqüente no grupo Osteoporose do que nos demais; e a menopausa, sendo que $100 \%$ das mulheres do grupo Osteoporose estavam na pós-menopausa, contra $86 \%$ das mulheres dos outros grupos. No histórico pessoal de fraturas, as do grupo Osteoporose relatavam maior número de fraturas prévias do que as demais e isso foi estatisticamente significante. A partir da identificação das principais

\begin{abstract}
Objectives: to assess the main risk factors associated to low bone mass and to propose algorithms for the indication of the evaluation of bone mineral density in a women population from São José do Rio Pardo, state of São Paulo. Methods: 324 women (mean age 60 years old) were studied. A questionnaire was sent by regular mail to collect data for several osteoporosis risk factors, as well as both personal and mother's history of fractures. Bone mineral density was measured using DXA, measuring the lumbar spine segment (L2-L4), and/or the proximal femur (femoral neck). Data were classified according to the WHO criteria. Data were analyzed using one-way Anova, and the "post-hoc" Student-Newman-Keuls test for paired multiple comparisons; $2 \times 2$ and $k x$ rontingency tables and chi-square test were also used. The most efficient variables for the identification of women at risk for osteoporosis were determined through multiple regression analysis. The previously described algorithms Osteorisk and Osiris were also applied. Results: there were 88 (27.2\%), 160 (49.4\%), and 76 (49.4\%) women among the normal, osteopenia, and osteoporosis groups, respectively. Mean age was 56, 60, and 65 years-old for the normal, osteopenia, and osteoporosis groups, respectively. Mean weight and height was 69.5 $\mathrm{kg} / 1.60 \mathrm{~m}, 64.9 \mathrm{~kg} / 1.59 \mathrm{~m}$ and $62.1 \mathrm{~kg} / 1.57 \mathrm{~m}$ for the normal, osteopenia, and osteoporosis groups, respectively. Differences between these mean values were statistically significant. The number of years of menopause was higher in the osteoporosis group; contraceptive and hormone replacement therapy was less frequent in the osteoporosis group, as well as menopause. All women in the osteoporosis group were post-menopause, compared to $86 \%$ in the other groups. Women in the osteoporosis group had more previous fractures, reaching statistical significance. Using
\end{abstract}

Serviço de Reumatologia do Hospital do Servidor Público Estadual - FMO, SP. Recebido em 25/5/2006. Aprovado, após revisão, em 06/1 1/06.

Disciplina de Reumatologia da Faculdade de Medicina da Universidade de Alfenas, MG.

1. Pós-graduando (mestrado) em Ciências da Saúde do Instituto de Assistência Médica ao Servidor Público Estadual - HSPE/FMO, SP. Professor da disciplina de Reumatologia da Faculdade de Medicina da Universidade de Alfenas, MG.

2. Diretor do Serviço de Reumatologia do Hospital do Servidor Público Estadual - Francisco Morato de Oliveira, SP.

Endereço para correspondência: Paulo Domingos Parisi Júnior, Rua Júlio de Mesquita, 577, CEP 13720-000, São José do Rio Pardo, SP, Brasil, telefone/fax (19) 36083146, e-mail: paulo@nosamed.com.br. 
variáveis, por meio de regressões múltiplas, chegou-se a uma equação envolvendo idade, peso e estatura que se mostrou o melhor modelo como preditor de osteoporose. Fez-se, então, uma comparação entre esse modelo, dois algoritmos descritos em literatura, Osteorisk e Osiris, e as variáveis idade e peso isoladamente. Conclusão: dentre as variáveis estudadas, idade e peso foram as que mostraram maior significância; sua combinação em um único algoritmo mostra desempenho melhor do que a utilização dessas variáveis individualmente. A inclusão de outras variáveis não melhora o desempenho na identificação de mulheres com osteoporose. A utilização de um algoritmo com critérios objetivos possibilita a seleção de candidatos à realização de densitometria óssea.

Palavras-chave: osteoporose, fatores de risco, densidade mineral óssea, mulheres.

\section{INTRODUÇÃO}

A presença de osteoporose está associada à fragilidade óssea aumentada e maior suscetibilidade à ocorrência de fraturas ${ }^{(1)}$. As fraturas, principalmente as de quadril, estão associadas a custos elevados, seja do ponto de vista de saúde, social ou econômico ${ }^{(2-6)}$. Assim, várias formas são propostas para identificar aquelas pessoas em risco e que poderiam se beneficiar de intervenções preventivas com o objetivo de se evitarem fraturas futuras, incluindo o uso de densitometria óssea como uma ferramenta diagnóstica e a prescrição de medicamentos para preservar a massa óssea ${ }^{(7)}$.

Existem evidências suficientes para que se possa afirmar que a densitometria óssea é, atualmente, o método mais efetivo para estimar o risco de fratura em mulheres na pós-menopausa ${ }^{(8)}$. Contudo, seu uso para rastreamento populacional, inclusive em mulheres após a menopausa, é controverso, em virtude de seu baixo poder para discriminar aqueles que irão fraturar daqueles que não o farão e de seu custo elevado, quando usado indiscriminadamente ${ }^{(9)}$.

Estabelecer critérios para selecionar indivíduos para a mensuração da densidade mineral óssea (DMO) é uma questão com importantes implicações econômicas ${ }^{(10)}$.

Várias tentativas têm sido feitas no sentido de desenvolver algoritmos para auxiliar na seleção de pacientes candidatos à realização de densitometria óssea, usandose uma variedade de combinações de fatores de risco. O propósito desses índices não é diagnosticar osteoporose ou osteopenia, mas identificar mulheres com maior probabilidade de apresentar fraturas, elegíveis para a realização de densitometria óssea ${ }^{(11-17)}$.

Associando-se os valores da DMO aos fatores de risco, busca-se melhorar a capacidade preditiva para risco de fraturas. Por outro lado, a avaliação dos fatores de risco auxilia sobremaneira na escolha dos potenciais candidatos à realização de densitometria óssea ${ }^{(18)}$. multiple regression, based on the main variables identified, we reached a formula considering age, weight, and height as the osteoporosis predicting model that was compared to the two algorithms Osteorisk and Osiris. Conclusion: among the variables studied, age and weight showed higher significance. Their combination in an algorithm showed better performance than either of the variables isolated. The inclusion of new variables did not increase the performance of the identification of osteoporotic women. The use of an algorithm with objective criteria makes it possible to better select candidates for bone densitometry analysis.

Keywords: osteoporosis, risk factors, bone mineral density, female-gender.

A densitometria óssea deve ser considerada quando ajudar na tomada de decisão a respeito de se estabelecer um tratamento medicamentoso para prevenção de fraturas por osteoporose. A decisão de se medir a DMO de determinado paciente deve levar em conta as evidências atuais de que o risco de fraturas aumenta com a idade e com a presença de um maior número de fatores de risco ${ }^{(9,19)}$.

Em nosso meio, a utilização rotineira de densitometria óssea pelo Sistema Único de Saúde (SUS) é coisa recente. A Portaria SAS/MS n ${ }^{\circ} 470$, de 23 de julho de 2002, estabelece o Protocolo Clínico e as Diretrizes Terapêuticas para Osteoporose. Essa Portaria visa à seleção de pacientes para o fornecimento de medicamentos para o tratamento da osteoporose. O principal critério de inclusão é a DMO igual ou inferior a $-2,5$ desvios-padrão do valor médio da massa óssea máxima para mulheres jovens normais, com ou sem fratura prévia. Portanto, para serem elegíveis ao tratamento, as pacientes necessariamente deverão realizar a densitometria óssea. Contudo, o número de exames densitométricos ofertados pelo SUS é relativamente pequeno. Levando-se em conta a cidade de São José do Rio Pardo, Estado de São Paulo, onde foi realizado o presente estudo, são 96 exames densitométricos anuais para uma população feminina acima de 50 anos de 5.273 (Dados do IBGE relativos ao ano de 2002). Por esses dados fica bastante clara a necessidade de uma escolha criteriosa dos pacientes que se submeterão à avaliação densitométrica.

A análise dos fatores de risco como preditores de baixa massa óssea assume grande importância na seleção dos candidatos ao exame densitométrico.

O objetivo do presente estudo foi verificar os principais fatores de risco associados à baixa massa óssea e propor algoritmos para a indicação de avaliação da densidade óssea em uma população de mulheres, residentes em São José do Rio Pardo, Estado de São Paulo. 


\section{PACIENTES E MÉTODO}

Foram selecionadas, por ordem alfabética, 1.200 mulheres, a partir do banco de dados de um serviço de densitometria óssea localizado em São José do Rio Pardo, cidade de 51 mil habitantes, situada a leste do Estado de São Paulo. A seleção tinha como alvo as mulheres sadias. Dessa forma, história de doenças malignas recentes ou outras condições de agravo à saúde que tivessem requerido longos períodos de imobilidade, além do uso de glicocorticóides, tornavam a mulher inelegível para este estudo.

Foi enviado, pelo correio, um questionário contendo perguntas a respeito de diversos fatores de risco para osteoporose e histórico pessoal e materno de fraturas.

Neste estudo, foram incluídas 324 mulheres que responderam ao questionário até a data da análise dos dados.

A densidade mineral óssea foi medida utilizando-se a densitometria óssea realizada por densitômetro DXA (dual energy X-ray absorptiometry) modelo DPX - IQ da marca Lunar. Foram analisados os sítios coluna lombar (L2-L4) e/ou fêmur proximal (colo femoral). Das 324 pessoas incluídas na pesquisa, 15 não realizaram a medição da $\mathrm{DMO}$ da coluna e 53 não o fizeram no fêmur proximal. A razão de números discrepantes de mensurações entre coluna e fềmur foi que, em determinadas situações, por razões de ordem operacional, foi medido apenas um segmento.

Foram utilizados os métodos estatísticos Análise de Variância (Anova), segundo um critério de classificação e teste "post-hoc" de comparação múltipla, aos pares, de Student - Newman - Keuls; tabelas de contigência 2 × 2 e k x r; teste de Qui-quadrado.

A partir dessas análises foram determinadas as variáveis mais significantes para baixa massa óssea. Realizando regressões múltiplas, foram estabelecidas as variáveis mais eficientes na identificação de mulheres com baixa massa óssea. Foram também aplicados em nossa amostra dois algoritmos descritos na literatura, denominados Osteorisk e Osiris (Osteoporosis Risk Index) ${ }^{(13,16)}$. Posteriormente, foram estabelecidos os pontos de corte, determinando sensibilidade, especificidade, valor preditivo positivo (VPP) e valor preditivo negativo (VPN).

\section{RESULTADOS}

A casuística, composta por 324 mulheres, apresentou a distribuição etária que está demonstrada na Figura 1.

$\mathrm{Na}$ Tabela $\mathrm{l}$, estão listadas as principais características da amostra em estudo.

No tocante à raça das 324 mulheres envolvidas no estudo, $294(90,74 \%)$ pertenciam à raça branca, 19 (5,86\%) classificaram-se como sendo pardas, nove $(2,78 \%)$ eram da raça negra e duas $(0,63 \%)$ da raça amarela.

Da casuística de 324 indivíduos, levando-se em consideração a classificação densitométrica da OMS para osteoporose, $88(27,2 \%)$ eram normais (grupo 1), $160(49,4 \%)$ apresentavam osteopenia (grupo 2$)$ e $76(23,5 \%)$ tinham osteoporose (grupo 3).

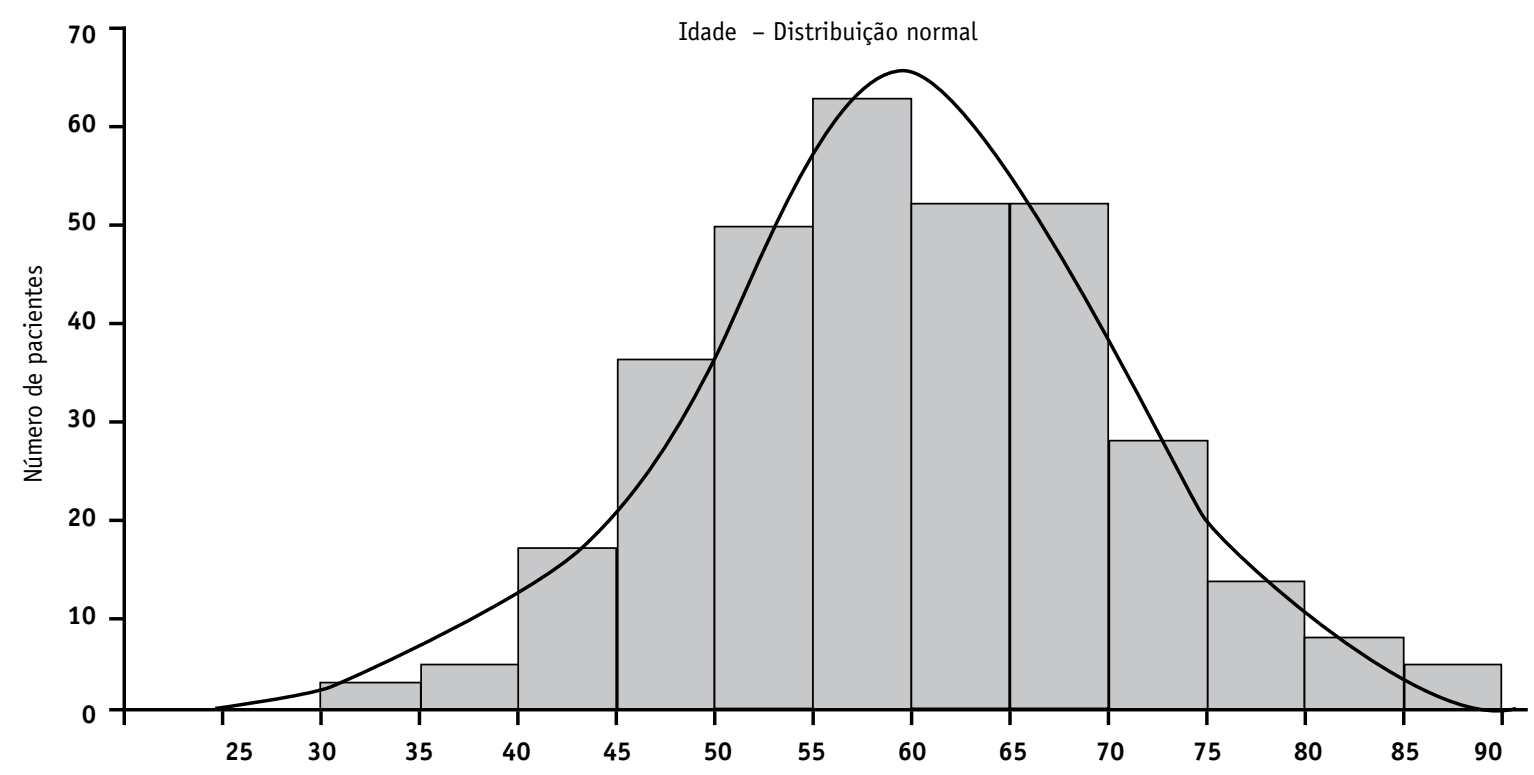

Figura 1 - Gráfico demonstrativo da distribuição etária dos casos. Kolmogorov-Smirnov - Dmax $=0,05449 p>0,20$. Distribuição normal da idade. 
TABELA 1

PRINCIPAIS CARACTERÍSTICAS DA AMOSTRA

\begin{tabular}{lcccc}
\hline Variável & N & Média & Desvio-padrão \\
\hline Idade (anos) & 324 & 60,12 & 9,96 \\
\hline Altura (m) & 324 & 1,59 & 0,06 \\
Peso (kg) & 324 & 65,49 & 11,87 \\
IMC & 324 & 26,04 & 4,45 \\
\hline Menarca (idade) & 306 & 13,07 & 1,63 \\
\hline Menopausa (idade) & 280 & 47,65 & 5,43 \\
Peso aos 25 anos (kg) & 276 & 53,34 & 7,56 \\
\hline Altura aos 25 anos (m) & 268 & 1,60 & 0,11 \\
\hline
\end{tabular}

Das variáveis estudadas, as que se mostraram estatisticamente significantes foram: idade, peso, estatura, índice de massa corporal (IMC) (Tabela 2), número de anos decorridos após a menopausa, peso relatado aos 25 anos de idade (Tabela 3), uso de anticoncepcionais e terapia de reposição hormonal (Tabela 4), histórico de fraturas nos membros inferiores e fraturas em qualquer sítio (Tabela 5).

TABELA 2

COMPARAÇÃO DAS VARIÁVEIS IDADE, PESO, ESTATURA E IMC NOS GRUPOS NORMAL, OSTEOPENIA E OSTEOPOROSE

\begin{tabular}{l|c|c|c}
\hline $\begin{array}{l}\text { Variáveis } \\
\text { N: 324 }\end{array}$ & $\begin{array}{c}\text { Normal } \\
\text { Média (dp) } \\
\text { N: 88 }\end{array}$ & $\begin{array}{c}\text { Osteopenia } \\
\text { Média (dp) } \\
\text { N: 160 }\end{array}$ & $\begin{array}{c}\text { Osteoporose } \\
\text { Média (dp) } \\
\text { N: 76 }\end{array}$ \\
\hline Idade (anos) & $56,24(+/-8,8)$ & $59,80(+/-9,8)$ & $65,48(+/-9,5)^{* * * *}$ \\
\hline Peso (kg) & $69,50(+/-13,6)$ & $64,87(+/-11,1)$ & $62,14(+/-9,9)^{* * *}$ \\
Estatura (m) & $1,60(+/-0,06)$ & $1,59(+/-0,06)$ & $1,57(+/-0,07)^{* *}$ \\
IMC & $27,02(+/-4,98)$ & $25,82(+/-4,33)$ & $25,34(+/-3,85)^{*}$ \\
\hline Anova $-{ }^{*} p<0,05{ }^{* *} p<0,005$ & $* * * p<0,0005{ }^{* * *} p<0,00001$
\end{tabular}

TABELA 3

COMPARAÇÃo dAS VARIÁVEIS NÚMERO DE ANOS DECORRIDOS DESDE A MENOPAUSA E PESO AOS 25 ANOS NOS GRUPOS NORMAL, OSTEOPENIA E OSTEOPOROSE

\begin{tabular}{c|c|c|c}
\hline Variáveis & $\begin{array}{c}\text { Normal } \\
\text { Média (dp) }\end{array}$ & $\begin{array}{c}\text { Osteopenia } \\
\text { Média (dp) }\end{array}$ & $\begin{array}{c}\text { Osteoporose } \\
\text { Média (dp) }\end{array}$ \\
\hline Anos após menopausa & $11,75(+/-8,45)$ & $13,38(+/-8,39)$ & $18,04(+/-10,15)^{\star * *}$ \\
N: 280 & N: 65 & N: 141 & N: 74 \\
Peso aos 25 anos & $55(+/-7,82)$ & $53,8(+/-7,75)$ & $51(+/-6,41)^{* *}$ \\
N: 276 & N: 68 & N: 134 & N: 74 \\
\hline
\end{tabular}

Anova $-{ }^{* *} p<0,005 \quad * * * p<0,0005$

\section{TABELA 4}

DisTRIBUiÇÃo dOS CASOS PELO USO DE ANTICONCEPCIONAIS E TRH E SUA CLASSIFICAÇÃO NOS GRUPOS (NORMAL + OSTEOPENIA E OSTEOPOROSE)

$\mathrm{N}: 324$

\begin{tabular}{lcc}
\hline Variáveis & Grupo normal + osteopenia & Grupo osteoporose \\
\hline Uso de anticoncepcionais & $52,83 \%^{*}$ & $35,53 \%^{*}$ \\
Uso de TRH & $60,20 \% \%^{* *}$ & $40,55 \% \%^{* *}$ \\
\hline
\end{tabular}

Qui-quadrado $-{ }^{*} p<0,05{ }^{* *} p<0,01$

Rev Bras Reumatol, v. 47, n.1, p. 16-24, jan/fev, 2007
TABELA 5

DisTRIBUIÇÃO DOS CASOS PELA INCIDÊNCIA DE FRATURAS NOS MEMBROS INFERIORES E EM QUALQUER SÍTIO E SUA CLASSIFICAÇÃO NOS GRUPOS

( 1 - NORMAL, 2 - OSTEOPENIA, 3 - OSTEOPOROSE) $\mathrm{N}: 324$

\begin{tabular}{lcccc}
\hline Variáveis & Normal & Osteopenia & Osteoporose \\
\hline Fraturas de membros inferiores & $5,5 \%^{*}$ & $14,4 \%^{*}$ & $18,4 \%^{*}$ \\
Fraturas em qualquer sítio & $13,6 \%^{* *}$ & $26,2 \%{ }^{* *}$ & $38,2 \%{ }^{* *}$ \\
\hline
\end{tabular}

Qui-quadrado - * $p<0,05{ }^{* *} p=0,001$

A idade da menopausa não mostrou diferença nos três grupos estudados, contudo, foi significante o fato de $100 \%$ das mulheres do grupo 3 (Osteoporose) já estarem na menopausa $(\mathrm{p}=0,0001)$, contra $86 \%$ das mulheres dos outros grupos.

A partir desses dados, fazendo regressões múltiplas, foram elaboradas equações testando-se diversos modelos, envolvendo as principais variáveis. Dos diversos modelos, a melhor equação foi a que envolveu idade, estatura e peso corporal (Tabelas 6 e 7).

TABELA 6

COEFICIENTES DE REGRESSÃO PARA AS VARIÁVEIS DO MODELO FINAL

\begin{tabular}{lcccc}
\hline Variáveis & Valor estimado & Erro-padrão & $\mathbf{p}$ \\
\hline Intercept & 3,1626 & 1,0029 & 0,001766 \\
Idade & 0,0224 & 0,0037 & 0,000000 \\
Estatura & $-1,1191$ & 0,6333 & 0,078190 \\
Peso & $-0,0118$ & 0,0033 & 0,000376 \\
\hline
\end{tabular}

Equação: grupos 1, 2 ou $3=3,1626+0,0224$ (idade) $-1,1191$ (estatura) $-0,0118$ (peso).

$r=0,40527 \quad N=324 \quad p=0,000001$

TABELA 7

COMPARAÇÃO DO RESULTADO DA EQUAÇÃO OBTIDA POR MEIO DE REGRESSÕES MÚLTIPLAS (TABELA 5 ) NOS GRUPOS ( 1 - NORMAL, 2 - OSTEOPENIA, 3 - OSTEOPOROSE) $\mathrm{N}: 324$

\begin{tabular}{l|c|c|c}
\hline Grupos & $\mathbf{N}$ & $\begin{array}{c}\text { Equação } \\
\text { (média) }\end{array}$ & $\begin{array}{c}\text { Equação } \\
\text { (desvio-padrão) }\end{array}$ \\
\hline Grupo 1 & 88 & $1,81^{*}$ & 0,23 \\
Grupo 2 & 160 & $1,96^{*}$ & 0,28 \\
Grupo 3 & 76 & $2,14^{*}$ & 0,28 \\
\hline
\end{tabular}

Anova - ${ }^{*} p=0,0000001$

Equação: grupos 1, 2 ou $3=3,1626+0,0224$ (idade) $-1,1191$ (estatura) $-0,0118$ (peso).

Aplicando-se a equação descrita acima na amostra estudada, os valores variaram de 1,18 a 2,86. Foi possível estabelecer um ponto de corte para a identificação de mulheres com osteoporose no valor 1,87 que conferiu uma 
sensibilidade de $80,3 \%$ e especificidade de $42,3 \%$. De modo arbitrário, dividiu-se a casuística em três grupos de risco para osteoporose, levando-se em consideração os valores obtidos pela aplicação da equação. Mulheres com valores iguais ou acima de 2,25 foram consideradas de alto risco para osteoporose; nesse grupo, que representa $17,6 \%$ da amostra, a prevalência de osteoporose foi de $52,6 \%$. Os casos com valores abaixo de 1,87 foram considerados de baixo risco, representam $37 \%$ dos casos e apenas $12,5 \%$ apresentam osteoporose. No grupo intermediário (valores iguais ou acima de 1,87 e abaixo de 2,25 ), que representa $45,4 \%$ dos casos, a prevalência de osteoporose foi de $21,1 \%$.

Com os dados obtidos nessa casuística, foi possível a aplicação de dois algoritmos descritos em literatura, usados para identificar mulheres com maior probabilidade de terem baixa DMO, a saber, Osteorisk e Osiris (Osteoporosis Risk Index $)^{(13,16)}$.

Aplicando o algoritmo Osteorisk (subtrai-se a idade do peso, multiplica-se por 0,2 e despreza-se o decimal) em nossa população de 324 mulheres, os valores variaram de -7 a $+9,6$. O ponto de corte foi o índice " 1 ", conferindo $80 \%$ de sensibilidade e $46 \%$ de especificidade na identificação de mulheres com osteoporose. Dividindo-se a amostra em três categorias de risco para osteoporose, a categoria de baixo risco (Osteorisk >1) representa 40\% da casuística e somente 11,6\% nessa categoria apresenta osteoporose (DMO T-score $<-2,5)$ em pelo menos um dos dois sítios estudados. A prevalência de osteoporose foi de $51 \%$ no grupo de alto risco (Osteorisk < ou $=-2$, representando $17 \%$ da amostra). No grupo de risco intermediário $(-2<$ Osteorisk $<+1)$, que representa $43 \%$ da população, a prevalência de osteoporose foi de $23,6 \%$.

Da mesma forma, foi utilizado o algoritmo Osiris (subtrai-se a idade do peso, multiplica-se por 0,2 , despreza-se o decimal e somam-se os valores; se fizer uso de terapia de reposição hormonal, acrescenta-se 2 ao resultado, se houver história de fratura de baixo impacto, subtrai-se 2). Os valores encontrados variaram de $-8,8$ a $10,4 \mathrm{em}$ uma amostra de 282 mulheres. O ponto de corte foi o índice "l", mostrando $74,3 \%$ de sensibilidade e $52,9 \%$ de especificidade. A amostra foi dividida em três categorias, a saber, alto, médio e baixo risco. A categoria de baixo risco (Osiris $>1$ ) representa $46 \%$ da casuística, e somente $14,7 \%$ nessa categoria apresentam osteoporose (DMO T-score $<-2,5) \mathrm{em}$ pelo menos um dos dois sítios estudados. A prevalência de osteoporose foi de $54 \%$ no grupo de alto risco (Osiris < ou $=-3$, representando $13,1 \%$ da amostra). No grupo de risco intermediário $(-3<$ Osiris $<+1)$, que representa $41 \%$ da população, a prevalência de osteoporose foi de $30,2 \%$.
Comparamos, então, o desempenho dos índices Osteorisk e Osiris, aplicados em nossa casuística, com o modelo envolvendo idade, estatura e peso, e também com as variáveis idade e peso isoladamente.

Nas Tabelas 8 e 9, estão listados sensibilidade, especificidade, valor preditivo positivo (VPP) e valor preditivo negativo (VPN) dos algoritmos Osteorisk e Osiris; da equação envolvendo idade, peso e estatura; do peso e da idade de forma isolada, em dois pontos de corte distintos, de acordo com maior sensibilidade e especificidade respectivamente.

TABELA 8

COMPARAÇÃO DA SENSIBILIDADE, ESPECIFICIDADE, VALOR PREDITIVO POSITIVO (VPP) E VALOR PREDITIVO NEGATIVO

(VPN) OBTIDOS NA APLICAÇÃO DOS ALGORITMOS E DAS PRINCIPAIS VARIÁVEIS ISOLADAMENTE. VALOR DE CORTE CONFERINDO MAIOR SENSIBILIDADE

\begin{tabular}{l|c|c|c|c|c}
\hline Índice & $\begin{array}{c}\text { Valor de } \\
\text { corte }\end{array}$ & $\begin{array}{c}\text { Sensibilidade } \\
\%\end{array}$ & $\begin{array}{c}\text { Especificidade } \\
\%\end{array}$ & VPP \% & $\begin{array}{c}\text { VPN } \\
\%\end{array}$ \\
\hline Osteorisk & $<$ ou $=1$ & 80,3 & 45,9 & 31,3 & 88,4 \\
\hline Osiris & $<$ ou $=1$ & 74,3 & 52,9 & 36 & 85,3 \\
$\begin{array}{l}\text { Equação } \\
\text { (IPE) }\end{array}$ & ou = 1,87 & 80,3 & 42,3 & 29,9 & 87,5 \\
Idade & $>55$ anos & 82,9 & 38,7 & 29,3 & 88,1 \\
Peso & $<73 \mathrm{~kg}$ & 86,8 & 29,5 & 27,4 & 88 \\
\hline
\end{tabular}

IPE - equação envolvendo as variáveis idade, peso e estatura.

TABELA 9

COMPARAÇÃO DA SENSIBILIDADE, ESPECIFICIDADE, VALOR PREDITIVO POSITIVO (VPP) E VALOR PREDITIVO NEGATIVO

(VPN) OBTIDOS NA APLICAÇ̃̃O DOS ALGORITMOS E DAS PRINCIPAIS VARIÁVEIS ISOLADAMENTE.

VALOR DE CORTE CONFERINDO MAIOR ESPECIFICIDADE

\begin{tabular}{l|c|c|c|c|c}
\hline Índice & $\begin{array}{c}\text { Valor de } \\
\text { corte }\end{array}$ & $\begin{array}{c}\text { Sensibilidade } \\
\%\end{array}$ & $\begin{array}{c}\text { Especificidade } \\
\%\end{array}$ & $\begin{array}{c}\text { VPP } \\
\%\end{array}$ & $\begin{array}{c}\text { VPN } \\
\%\end{array}$ \\
\hline Osteorisk & $<$ ou $=-2$ & 36,8 & 89,1 & 50,9 & 82,2 \\
\hline Osiris & $<$ ou $=-3$ & 27 & 91,8 & 54,1 & 78 \\
\hline $\begin{array}{l}\text { Equação } \\
\text { IPE }\end{array}$ & ou $=2,25$ & 39,5 & 89,1 & 52,6 & 82,8 \\
Idade & $>70$ anos & 35,5 & 91,1 & 55,1 & 82,2 \\
Peso & $<$ ou = 57 kg & 31,6 & 75,8 & 28,6 & 78,3 \\
\hline
\end{tabular}

IPE - equação envolvendo as variáveis idade, peso e estatura.

Se dividirmos a casuística em três grupos para cada índice testado, de acordo com os valores de corte descritos acima, denominando-os de grupos de alto, médio e baixo risco para osteoporose, obtivemos os resultados apresentados nas Tabelas 10 e 11 .

Essa divisão possibilita selecionar uma parte de menos de $20 \%$ da amostra cuja prevalência de osteoporose ultrapassa $50 \%$. Da mesma forma, para o grupo de baixo 
TABELA 10

COMPARAÇÃO DO PORCENTUAL DE INDIVÍDUOS EM RELAÇÃO AO TOTAL DA CASUÍSTICA E O DE PORTADORES DE OSTEOPOROSE OBTIDO NA APLICAÇÃO DOS ALGORITMOS E DAS PRINCIPAIS VARIÁVEIS ISOLADAMENTE. GRUPO DE ALTO RISCO PARA OSTEOPOROSE

\begin{tabular}{lcccc}
\hline Índice & Valor de corte & \% da casuística & \% com osteoporose \\
\hline Osteorisk & $<\mathrm{ou}=-2$ & 17 & 51 \\
Osiris & $<\mathrm{ou}=-3$ & 13,1 & 54 \\
Equação IPE & $>\mathrm{ou}=2,25$ & 17,6 & 52,6 \\
Idade & $>70$ anos & 15,1 & 55,1 \\
Peso & $<\mathrm{ou}=57 \mathrm{~kg}$ & 25 & 29,6 \\
\hline
\end{tabular}

IPE - equação envolvendo as variáveis idade, peso e estatura.

TABELA 11

Comparaç̃o do PORCENTUAL de INDIVÍdUUOS EM RELAÇÃO AO TOTAL DA CASUÍSTICA E O DE PORTADORES DE OSTEOPOROSE OBTIDO NA APLICAÇÃO DOS ALGORITMOS E DAS PRINCIPAIS VARIÁVEIS ISOLADAMENTE. GRUPO DE BAIXO RISCO PARA OSTEOPOROSE

\begin{tabular}{lccccc}
\hline Índice & Valor de corte & \% da casuística & \% com osteoporose \\
\hline Osteorisk & $>1$ & 40 & 11,6 \\
Osiris & $>1$ & 46 & 14,7 \\
Equação IPE & $<1,87$ & 37 & 12,5 \\
Idade & $<\mathrm{ou}=55$ anos & 33,6 & 11,9 \\
Peso & $>\mathrm{ou}=73 \mathrm{~kg}$ & 25 & 11,1 \\
\hline
\end{tabular}

IPE - equação envolvendo as variáveis idade, peso e estatura.

risco, selecionamos uma fatia de aproximadamente $40 \%$ da amostra que contém pouco mais de $10 \%$ de indivíduos com osteoporose, independentemente do índice por nós utilizado. Assim, verificamos que todos os índices apresentaram desempenho semelhante, com exceção do peso, isoladamente, que na linha de corte de maior especificidade para osteoporose mostrou um VPP de $28,6 \%$, enquanto os demais índices, nessa mesma linha de corte, mostraram VPP acima de 50\% (Tabela 9).

No tocante à sensibilidade, para selecionar os candidatos à realização de densitometria óssea, verificamos que Osteorisk $(<$ ou $=1)$ seleciona $60 \%$ da casuística e, destes, $31,3 \%$ apresentam osteoporose; Osiris ( $<$ ou = 1) seleciona $54 \%$ da casuística e, destes, 36\% apresentam osteoporose; a equação, envolvendo três variáveis (> ou $=1,87$ ) seleciona $63 \%$ da casuística e, destes, 29,9\% apresentam osteoporose; a idade (> 55 anos) seleciona 66,4\% da casuística e, destes, 29,3\% apresentam osteoporose; e, por fim, o peso $(<73 \mathrm{~kg})$ seleciona $75 \%$ da casuística e, destes, $27,4 \%$ apresentam osteoporose.

Verificou-se também uma significante correlação da idade (inversamente proporcional) e peso (diretamente proporcional) com a DMO dos segmentos L2-L4 e colo femoral. Dessa forma, existe uma correlação entre maior risco para osteoporose e t escore. Independentemente do índice utilizado, no grupo de alto risco para osteoporose, cerca de $70 \%$ das mulheres apresentam t escore menor ou igual a -2,0 em pelo menos um dos dois sítios estudados.

\section{DISCUSSÃO}

As sugestões relativas a quem deve ou não se submeter à mensuração da DMO são muitas. As recomendações da National Osteoporosis Foundation (NOF) indicam a realização de densitometria óssea em mulheres com 65 anos ou mais e em mulheres mais novas, na pós-menopausa, que tenham um ou mais fatores de risco para fratura por osteoporose além da menopausa ${ }^{(20)}$.

Esse tipo de recomendação que seleciona mulheres na perimenopausa, baseando-se em "outros fatores de risco", é bastante freqüente e está presente, por exemplo, nas recomendações da Osteoporosis Society of Canada (OSC), da International Society for Clinical Densitometry (ISCD) e também, em nosso meio, no Protocolo Clínico e Diretrizes Terapêuticas para Osteoporose do Ministério da Saúde ${ }^{(21,22)}$.

Contudo, dado que a maioria das mulheres na pós-menopausa apresenta pelo menos um desses fatores de risco, a questão muitas vezes passa a ser não em quem realizar o exame, mas, sim, em quem não fazer o exame ${ }^{(18)}$.

Uma revisão sistemática de literatura envolvendo 94 estudos coorte e 72 estudos caso-controle revelou cerca de 80 fatores que podem estar relacionados com risco de fraturas por perda de massa óssea. Dos fatores de risco para fraturas relacionados à perda de massa óssea identificados, $15 \%$ foram classificados como de alto risco $(R R>o u=a 2)$, $18 \%$ de risco moderado ( $R R$ entre 1 e 2 ) e $8 \%$ mostraram um risco relativo próximo a 1 , sendo classificados como sem risco. Cinqüenta e nove por cento dos fatores de risco identificados não puderam ser classificados em nenhum dos grupos descritos acima, seja por falta de informações ou pelos resultados contraditórios nos diversos estudos. Os fatores classificados como de alto risco foram: idade (maior que 70 anos), baixo peso (IMC inferior a $20 \mathrm{~kg} / \mathrm{m}^{2}$ ou peso inferior a $40 \mathrm{~kg}$ ), perda de peso a partir dos 25 anos (maior que $10 \%$ ), inatividade física, uso de corticosteróides, fratura prévia por osteoporose, uso de anticonvulsivantes, hiperparatireoidismo primário, diabetes melito tipo I, anorexia nervosa, gastrectomia e anemia perniciosa. Como médio risco estão incluídos: sexo feminino, tabagismo atual, baixa exposição ao sol, história familiar de fratura osteoporótica, 
menopausa cirúrgica, menopausa precoce (antes dos 45 anos), menarca tardia (após os 15 anos), período fértil curto (menor que 30 anos), não ter amamentado, baixa ingesta de cálcio, hipertireoidismo e artrite reumatóide. O consumo de cafeína, chá, água fluorada e diuréticos tiazídicos, assim como a nuliparidade, foram classificados como sem risco ${ }^{(10)}$.

Esses dados ilustram a dificuldade da utilização de fatores de risco de forma racional para a indicação da mensuração da DMO.

Assim, algoritmos simples para seleção de candidatos à mensuração da DMO podem auxiliar a reduzir as dúvidas na prática clínica, ao introduzir critérios mais claros para o uso dos principais fatores de risco.

Em todos os algoritmos pesquisados, duas variáveis estão sempre presentes, que são idade e peso. Esse fato, por si só, já é indicativo da importância desses fatores de risco como preditores para osteoporose ${ }^{(23-26)}$. Outras variáveis presentes na maioria dos algoritmos foram fraturas prévias de baixo impacto e terapia estrogênica. Em nossa casuística, essas variáveis, juntamente com estatura atual e peso relatado aos 25 anos, foram as que apresentaram maior significância estatística.

Tais algoritmos não identificam todos os casos de osteoporose, mas podem ajudar a aumentar a eficiência da densitometria óssea na identificação dos pacientes com osteoporose.

Estão relacionados a seguir os principais algoritmos propostos para essa finalidade: Simple Calculated Osteoporosis Risk Estimation (Score): os fatores de risco envolvidos são raça, presença de artrite reumatóide, presença de fraturas não-traumáticas, idade, terapia estrogênica e peso ${ }^{(11)}$; Osteoporosis Self-assessment Tool (OST), ou Female Osteoporosis Self-assessment Tool for Asians (Fosta), ou Osteorisk (para a América Latina): mesmo algoritmo, cuja denominação varia dependendo da população estudada, os fatores de risco utilizados são peso e idade ${ }^{(12,13)}$; Osteoporosis Risk Assessment Instrument (Orai): leva em conta a idade, peso e uso de estrógeno ${ }^{(14)}$; Study of Osteoporotic Fractures, Simple, Useful Risk Factor System (Sofsurf): utiliza idade, peso, tabagismo e história de fraturas na pós-menopausa ${ }^{(15)}$; Osteoporosis Index of Risk (Osiris): considera os fatores peso, idade, uso de estrógenos e história de fraturas de baixo impacto ${ }^{(16)}$; Abone (A: age, B: bulk, One: or never estrogens): utiliza as variáveis idade, peso e uso de estrógeno (contraceptivos orais ou terapia de reposição hormonal) ${ }^{(17)}$.

Todos esses índices mostraram-se eficientes na identificação de mulheres com osteoporose, com sensibilidade variando entre $78,5 \%$ e $94 \%$ e especificidade de $41,4 \%$ a $51,4 \%$, números semelhantes aos por nós encontrados.

Alguns desses instrumentos utilizam um único ponto de corte, de forma dicotômica, estratificando as mulheres em alto risco para osteoporose (deve ser medida a DMO) e baixo risco (não deve ser medida a DMO); outros optam pelo uso de dois pontos de corte, dividindo a população em três categorias, classificando em baixo, moderado e alto risco.

Nossa estratégia envolveu a divisão em três categorias, que nos parece uma alternativa mais útil do que um único ponto de corte.

Dos índices por nós testados, aqueles envolvendo uma única variável, ou seja, idade e peso isoladamente, são passíveis de sofrer maior influência da amostra populacional envolvida, dependendo da faixa etária e ponderal. A combinação das duas variáveis apresenta um desempenho melhor e está menos sujeita a essas influências ${ }^{(12)}$.

A alternativa da inclusão de uma terceira variável, no caso a estatura, em nada modificou o desempenho na identificação de pacientes com osteoporose.

$\mathrm{O}$ algoritmo Osiris, que combina idade, peso, uso de terapia de reposição hormonal e história prévia de fratura, tem maior especificidade, porém menor sensibilidade quando comparado ao Osteorisk, que utiliza apenas idade e peso. Assim, Osteorisk nos parece melhor, em virtude de ter maior sensibilidade e envolver um número menor de variáveis, o que facilita sua aplicação.

O algoritmo Osteorisk foi elaborado a partir de uma amostra populacional latino-americana com 1.547 mulheres na pós-menopausa, acima de 50 anos, envolvendo vários países, com a participação do Brasil (16,9\%). A correlação foi feita com a DMO do colo femoral. Nessa população, o ponto de corte foi o índice "l", determinando uma sensibilidade de $92 \%$ e especificidade de $45 \%$. A média de idade foi 64,6 anos, e a porcentagem da amostra com osteoporose foi de $32,5 \%$; a prevalência de osteoporose foi de $66 \%$ no grupo de alto risco e de $8 \%$ no grupo de baixo risco. Nossos resultados, quando da aplicação do algoritmo Osteorisk, mostraram-se mais discretos, principalmente no que diz respeito à sensibilidade. Uma provável causa talvez seja a diferença na faixa etária, que inclusive se refletiu na porcentagem global de osteoporose, maior no trabalho original. Também o tamanho da casuística deve ser levado em consideração, juntamente ao fato de, em nossa amostra, não selecionarmos somente mulheres na pós-menopausa, com mais de 50 anos. Contudo, nos aspectos principais os resultados foram semelhantes e os pontos de corte foram 
os mesmos. Em ambos os trabalhos, no grupo de alto risco, a prevalência de casos com osteoporose foi praticamente $o$ dobro do que na amostra como um todo ${ }^{(13)}$.

A utilização de três categorias de risco possibilita sugerir uma estratégia de indicação para a mensuração da DMO. Nos parece razoável recomendar o tratamento de mulheres do grupo de alto risco mesmo sem medir a DMO, especialmente para mulheres com fatores de risco adicionais, pois aproximadamente metade ou mais delas apresenta osteoporose e cerca de $70 \%$ têm $\mathrm{t}$ escore menor ou igual a -2 . No outro extremo, um grupo de baixo risco é identificado, a prevalência de osteoporose nesse grupo é de aproximadamente $10 \%$. É possível postergar a mensuração da DMO nessas mulheres a menos que tenham outros fatores de risco como, por exemplo, uso de corticosteróides. Isso representaria uma economia considerável, visto que esse grupo perfaz uma grande proporção de mulheres na pós-menopausa, cerca de $40 \%$. Para o grupo intermediário, a mensuração da DMO é provavelmente necessária para identificar os indivíduos com osteoporose, pois a prevalência de casos não possibilita tomada de decisões relativas a tratamento.

À primeira vista pode parecer estranho que um índice apresente bom desempenho usando apenas duas variáveis (idade e peso). Contudo, existe uma grande amplitude de valores para idade e peso, enquanto outras variáveis contribuem somente com 1 ou 2 pontos para os índices. Além disso, todas as mulheres certamente têm peso e idade, enquanto outros fatores de risco estarão presentes em apenas alguns subgrupos. De fato, o aumento da idade e o decréscimo de peso têm sido associados à baixa DMO e ao aumento do risco de fraturas ${ }^{(23,26)}$. Um estudo realizado no Canadá com 926 mulheres na pós-menopausa chegou a um índice baseado em idade, peso corporal e uso atual de estrógeno. O uso do estrógeno contribuiu com 2 pontos, enquanto a idade contribuiu com até 15 pontos e o peso com até 9 pontos. Esse índice, denominado Osteoporosis

\section{REFERÊNCIAS}

1. Nordin BEC: The definition and diagnosis of osteporosis. Calcif Tissue Int 40: 57-8, 1987.

2. Riggs BL, Melton LJ III: The worldwide problem of osteoporosis: insights afforded by epidemiology. Bone 17(5)(Suppl.): 505S11S, 1995.

3. Melton LJ III: How many women have osteoporosis now? J Bone Miner Res 10: 175-6, 1995.

4. Melton LJ III, Chrischilles EA, Cooper C, Lane AW, Riggs BL: Perspective: how many women have osteoporosis? J Bone Miner Res 7: 1005-10, 1992.
Risk Assessment Index (Orai), apresentou sensibilidade de $94 \%$ e especificidade de $41 \%$ na identificação de mulheres com osteoporose ${ }^{(14)}$.

Em um estudo multicêntrico envolvendo 32.515 mulheres na pós-menopausa com 45 anos ou mais, comparou-se a capacidade de quatro índices já publicados para identificar mulheres com baixa densidade óssea. A sensibilidade e especificidade para identificar mulheres com osteoporose ficou, respectivamente, em $88 \%$ e $52 \%$ para o Osteoporosis Self-assessment Tool(OST), 90\% e 52\% para o Osteoporosis Risk Assessment Index (Orai), 89\% e 58\% para o Simple Calculated Osteoporosis Risk Estimation (Score) e 92\% e 37\% para o Study of Osteoporotic Fractures Simple Useful Risk Factor System (Sofsurf). A prevalência de osteoporose na categoria de baixo risco variou de $2 \%$ a $4 \%$ e na de alto risco de $47 \%$ a $61 \%$. A conclusão foi que todos os índices testados apresentaram desempenho semelhante e que o índice OST, pela sua simplicidade, pode ser útil na prática clínica ${ }^{(27)}$.

Outro estudo realizado no Japão com 1.127 mulheres na pós-menopausa com 47 anos ou mais comparou os índices Female Osteoporosis Self-assessment Tool for Asians (Fosta), Orai, Score e Sofsurf.

A sensibilidade para identificar mulheres com osteoporose variou de $87 \%$ a $90 \%$ e a especificidade foi de $38 \%$ a $43 \%$, conferindo um desempenho semelhante entre os índices ${ }^{(28)}$.

Concluindo, dentre as variáveis estudadas, idade e peso foram as que mostraram maior significância; sua combinação em um único algoritmo mostra desempenho melhor do que a utilização dessas variáveis individualmente, e a inclusão de outras variáveis não melhora o desempenho na identificação de mulheres com osteoporose. A utilização de um algoritmo com critérios objetivos possibilita a seleção de candidatos à realização de densitometria óssea.

Declaramos a inexistência de conflitos de interesse. 
9. NIH Consensus development panel on osteoporosis prevention, diagnosis and therapy. Jama 285: 785-95, 2001.

10. Espallargues M, Sampietro-Colom L, Estrada MD, et al: Identifying bone-mass-related risk factors for fracture to guide bone densitometry measurements: a systematic review of the literature. Osteoporos Int 12: 811-22, 2001.

11. Lydick E, Cook K, Melton M, Stine R, Byrnes C: Development and validation of a simple questionnaire to facilitate identification of women likely to have low bone density. Am J Manag Care 4: 37-48, 1998.

12. Koh LK, Sedrine WB, Torralba TP, et al: A simple tool to identify asian women at increased risk of osteoporosis. Osteoporos Int 12: 699-705, 2001.

13. Sem SS, Rives VP, Messina OD, et al: A Risk Assessment Tool (OsteoRisk) For Identifying Latin American Women With Osteoporosis. J Gen Intern Med 20(3): 245-50, 2005.

14. Cadarette SM, Jaglal SB, Kreiger N, Mcisaac WI, Darlington GA, Tu JV: Development and validation of the Osteoporosis Risk Assessment Instrument to facilitate selection of women for bone densitometry. CMAJ 162: 1289-94, 2000.

15. Black DM, Palermo L, Pearson J, Abbott T, aJohnell O: A simple, useful risk factor system can identify the large majority of women with osteoporosis. Bone 23 (Suppl. 5): 605s, 1998.

16. Sedrine WB, Chevalier T, Zegels B, et al: Development and assessment of the Osteoporosis Index of Risk (Osiris) to facilitate selection of women for bone densitometry. Gynecol Endocrinol 16: 245-50, 2002.

17. Weinstein L, Ullery B: Identification of at-risk women for osteoporosis screening. Am J Obstet Gynecol 183: 547-9, 2000.

18. Cadarette SM, Jaglal SB, Murray TM, Mcisaac WJ, Joseph L, Brown JP: Evaluation of decison rules for referring women for bone densitometry by dual-energy X-ray absorptiometry. Jama 286: 57-63, 2001.
19. Brown JP, Josse RG, for the Scientifc Advisory Council of the Osteoporosis Society of Canada: 2002 clinical practice guidelines for the diagnosis and management of osteoporosis in Canada. CMAJ 167 (Suppl. 10): sl-s34, 2002.

20. National Osteoporosis Foundation. Physician's Guide to prevention and treatment of osteoporosis. Belle Mead NJ, Excerpta Medica Inc, 1999.

21. Leib ES, Lewiecki EM, Binkley N, Hamdy RC, International Society for Clinical Densitometry: Official positions of the International Society for Clinical Densitometry. J Clin Densitom 7(1): 1-6, 2004.

22. Scientific Advisory Board, Osteoporosis Society Of Canada: Clinical practice guidelines for the diagnosis and management of osteoporosis. CMAJ 155: 1113-33, 1996.

23. Hui LS, Slemenda WC, Johnston CC: Age and bone mass as predictors of fracture in a prospective study. J Clin Invest 81 : 1804-9, 1988

24. Nilas L, Christiansen C: Bone mass and its relationship to age and the menopause. J Clin Endocrinol Metab 65: 697-702, 1987.

25. Michaëlson K, Bergströn R, Mallmin H, Holmberg L, Wolk A, Ljunghall S: Screening for osteopenia and osteoporosis: selection by body composition. Osteoporos Int 6: 120-6, 1996.

26. Omland LM, Tell GS, Ofjord S, Skag A: Risk factors for low bone mineral density among a large group of Norwegian women with fractures. Eur J Epidemiol 16: 223-9, 2000.

27. Geusens P, Hochberg MC, Van Der Voort DJM, et al: Performance of risk indices for identifying low bone density in postmenopausal women. Mayo Clin Proc 77: 629-37, 2002.

28. Fujiwara S, Masunari N, Suzuke G, Ross PD: Performance of osteoporosis risk indices in a japanese population. Curr Ther Res Clin Exp 62: 586-94, 2001. 